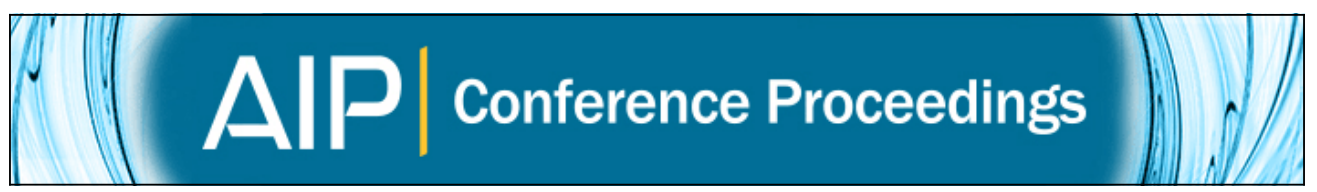

\title{
Star formation and dust obscuration at $\mathrm{z} \approx 2$
}

M. Pannella, C. L. Carilli, E. Daddi, H. J. Mc Cracken, F. Owen, A. Renzini, E. Schinnerer, and V. Smolčić

Citation: AIP Conference Proceedings 1111, 203 (2009); doi: 10.1063/1.3141544

View online: http://dx.doi.org/10.1063/1.3141544

View Table of Contents:

http://scitation.aip.org/content/aip/proceeding/aipcp/1111?ver=pdfcov

Published by the AIP Publishing

Articles you may be interested in

Physics of primordial star formation

AIP Conf. Proc. 1480, 43 (2012); 10.1063/1.4754326

Star Formation At High Redshift

AIP Conf. Proc. 783, 381 (2005); 10.1063/1.2035009

The nearby universe observed in far-infrared and in ultraviolet: an analysis of the dust attenuation and the star formation activity

AIP Conf. Proc. 761, 297 (2005); 10.1063/1.1913943

Cosmic star formation history

AIP Conf. Proc. 393, 481 (1997); 10.1063/1.52821

Current issues in star formation

AIP Conf. Proc. 393, 3 (1997); 10.1063/1.52807 


\title{
Star formation and dust obscuration at $\mathrm{z} \approx 2$
}

\author{
M. Pannella*, C. L. Carilli*, E. Daddi', H. J. Mc Cracken**, F. Owen*, \\ A. Renzini ${ }^{\ddagger}$, E. Schinnerer ${ }^{\S}$ and V. Smolčić \\ ${ }^{*}$ National Radio Astronomy Observatory, P.O. Box 0, Socorro, NM 87801-0387 \\ ${ }^{\dagger}$ CEA, Orme des Merisiers, F-91191 Gif-sur-Yvette, France \\ ${ }^{* *}$ Institut d'Astrophysique de Paris, 98 bis Boulevard Arago, 75014 Paris, France \\ $¥$ INAF - Osservatorio Astronomico di Padova, Vicolo dell'Osservatorio 5, I-35122 Padova, Italy \\ ${ }^{\S}$ Max Planck Institut fiur Astronomie, Klonigstuhl 17, Heidelberg, D-69117, Germany \\ ${ }^{\top}$ California Institute of Technology, MS 105-24, Pasadena, CA 91125
}

\begin{abstract}
We present preliminary results of a study aimed to constrain the star formation rate and dust content of galaxies as a function of mass at $z \approx 2$, a crucial time for galaxy evolution: this is the cosmic epoch when most of the stars in the Universe are formed. We use a sample of star forming galaxies to perform a stacking analysis of their $1.4 \mathrm{GHz}$ radio continuum as a function of different stellar population properties. Dust unbiased star formation rates are retrieved from radio fluxes. The main results of this work are: i) Galaxies in our sample show no downsizing pattern: specific star formation rates are constant over more than 1 dex in mass and up to the highest stellar mass probed; ii) a model independent UV dust attenuation law is provided which can be used to retrieve UV corrected SFR for extensive samples and hence a dust free cosmic star formation rate density.
\end{abstract}

Keywords: galaxies: formation and evolution - galaxies: star formation - galaxies: dust attenuation PACS: 98.62.Ai,98.62.Ck,98.62.Lv,98.62.Qz

\section{INTRODUCTION}

How and when galaxies build up their stellar mass is still a major question for observational cosmology. While a general consensus has been reached in the last years on the evolution of the galaxy stellar mass function (e.g. [1]), $\Phi(\mathrm{M}, \mathrm{z})$, the evolution of the star formation as a function of stellar mass $\operatorname{SFR}(\mathrm{M}, \mathrm{z})$ still remains unclear and debated. UV-based star formation rates (SFR) suffer from poorly understood dust attenuation correction. IR-SFRs suffer from poor resolution of IR detectors. On the other hand, thanks to the observed radio-IR correlation [2], and to the arcsecond resolution achievable with interferometric observations, radio continuum turns out to be the best dust unbiased SFR tracer. This study aims to disclose new clues on the downsizing scenario [3] and its evolution during cosmic times. Recent studies, mainly based on UV derived SFR, suggest the persistence of the downsizing pattern, i.e the most intense star formation occurs in low mass galaxies, out to very high redshifts (e.g. [4]).

\section{DATA AND ANALYSIS}

For this work we used the VLA-COSMOS $1.4 \mathrm{GHz}$ mosaic (2 square degrees, $\mathrm{rms} \approx 10 \mu \mathrm{Jy}, 1.5$ " resolution [5]) in combination with deep imaging in B,z (Subaru, [6]) and Ks (CFHT, Mc Cracken et al. in preparation) bands. A dust-unbiased

\footnotetext{
CP1111, Probing Stellar Populations Out to the Distant Universe, Cefali 2008, Proceedings of the International Conference, edited by L. A. Antonelli, E. Brocato, M. Limongi, N. Menci, G. Raimondo, and A. Tornambè 10 2009 American Institute of Physics 978-0-7354-0648-3/09/\$25.00
} 

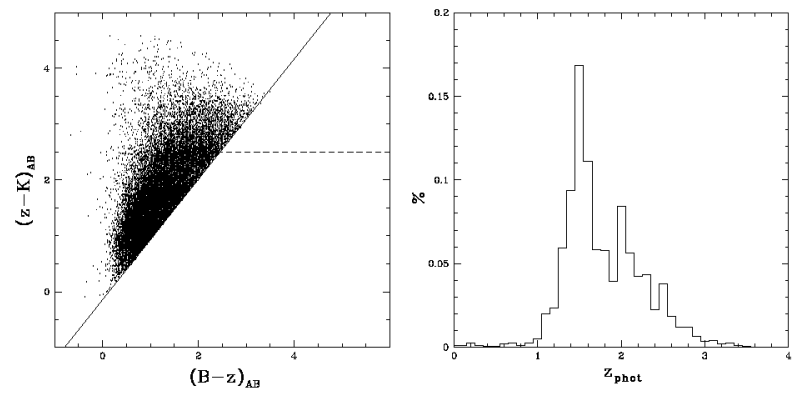

FIGURE 1. Left: Selection diagram for star forming galaxies at $z \approx 2$ according to [7]. Right: Photometric redshift distribution [10] of the sBzK sample: the bulk is in the redshift range $[1.3 \div 2.5]$.

sample of about 32000 star forming BzK galaxies (sBzK, see [7]), with $\mathrm{Ks}<23 \mathrm{AB}$ mag, was assembled to investigate, in a crucial redshift range for galaxy evolution studies, how star formation rate and dust attenuation depend on galaxy properties. We adopt the same recipes used in [7] to infer the stellar population properties of the sBzK galaxy sample. A Salpeter [8] initial mass function is used to derive stellar masses and SFRs.

About $2 \%$ of the $\mathrm{sBzK}$ catalog has a radio counterpart. However, these are mostly extreme objects: AGN or ULIRG-like starbursts [9]. In order to study star formation and dust content for the normal galaxy population, including objects undetected in the radio image, we used median stacking as a function of galaxy properties. Median stacking, compared to mean, is more robust against the tails of the distribution without altering the rms that still decreases as $\approx \mathrm{N}^{1 / 2}$. Total radio fluxes are retrieved by fitting a dirty beam convolved Gaussian function to the stacked data (Fig 2).

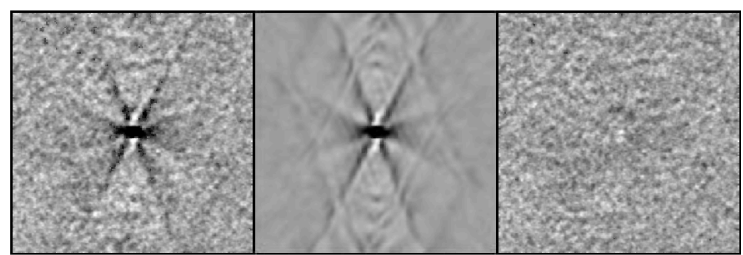

FIGURE 2. Left: Median stacking result for all the $32000 \mathrm{sBzK}$ galaxies. Middle: Best fit dirty beam convolved Gaussian function to the stacked data. The total flux recovered is $8.8 \pm 0.1 \mu \mathrm{Jy}$. Right: Residual.

\section{RADIO STACKING AS A FUNCTION OF GALAXY PROPERTIES}

Given the large number of objects in our sample, we can also stack the radio continuum as a function of different galaxy properties. In Fig. 3 we present our results for the radio stacking as a function of: $i$ ) the observed $\mathrm{B}$ band, i.e. the restframe dust uncorrected UV luminosity; ii) the (B-z) color which is a proxy, for galaxies at $\mathrm{z} \approx 2$, for the UV slope of the spectral energy distribution, i.e. for the dust/metals content; iii) the galaxy stellar 

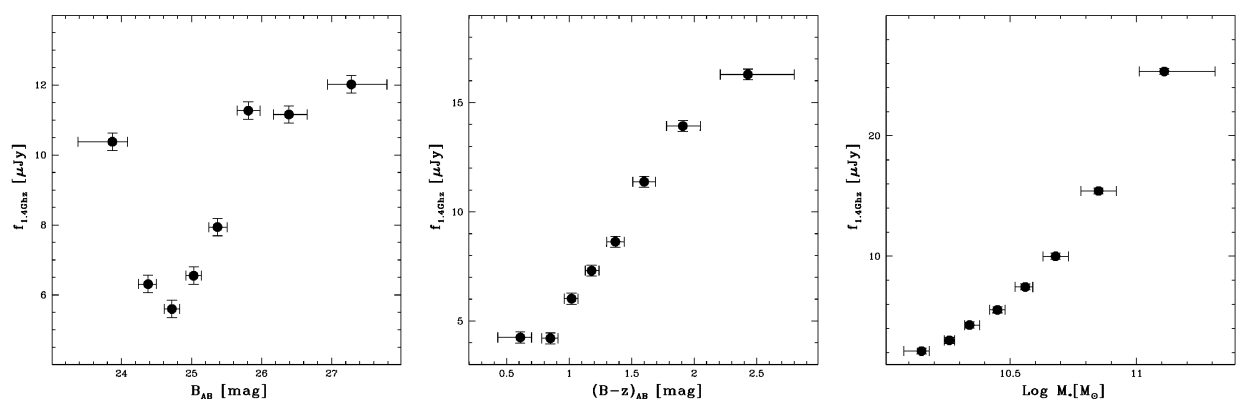

FIGURE 3. Radio stacking versus B band magnitude (left), B-z color (middle), stellar Mass (right).

mass. Assuming that radio flux can be used as a proxy for total star formation rate we conclude that: 1) the UV light, dust uncorrected, is poorly correlated with the ongoing star formation activity and counter intuitively: the largest SFRs happen in galaxies with the faintest UV light; 2) Galaxies with higher SFRs are more UV extincted; 3) Similarly to the local Universe, more massive galaxies show higher star formation activity.

\section{RADIO DERIVED SPECIFIC STAR FORMATION RATE}

Taking advantage of the high quality photometric redshifts available in the COSMOS survey [10] we can split our sBzK sample in two redshift bins centered at $\mathrm{z} \approx 1.5$ and 2.1 . We removed all X-ray detections in order to avoid any possible AGN contamination [11]. Using a synchrotron emission spectral index of -0.8 we $k$-correct radio fluxes in $1.4 \mathrm{GHz}$ luminosities. Adopting the conversion factor from the study of [2] we then convert radio luminosities in SFRs and finally derive specific star formation rates ( $\left.\mathrm{SSFR}=\mathrm{SFR} / \mathrm{M}_{*}\right)$. Additionally, deriving $\mathrm{UV}_{1500}$ luminosities, uncorrected by dust attenuation, from the observed B band magnitudes [7], we also compute UV-SSFR. We show our results in the left panel of Fig. 4: $i$ ) dust free SFRs show no downsizing in our data: SSFRs are constant over more than 1 dex in mass and up to the highest stellar mass probed (see also [12]); ii) by correcting the UV light with a single $\mathrm{A}_{1500}$ at all the masses, a solution often adopted in the literature, we would have produced an artificial, and wrong, downsizing pattern for this sample too; iii) more massive galaxies are more dust extincted.

\section{THE DUST ATTENUATION AT 1500Å}

Comparing the radio-SFRs with the uncorrected UV-SFRs as a function of galaxy stellar mass and (B-z) color, we are able to obtain a linear relation for the UV light attenuation by dust at $\mathrm{z} \approx 2$. [14] found a similar result for a sample of local starburst galaxies. Our relation naturally extends their results to higher redshifts. It also nicely shows the dust-unbiased nature of the BzK selection against, for example, the dropout technique where: "only galaxies with $A_{1500}<3.6 \mathrm{mag}$ are blue enough in the $U V$ to be selected 

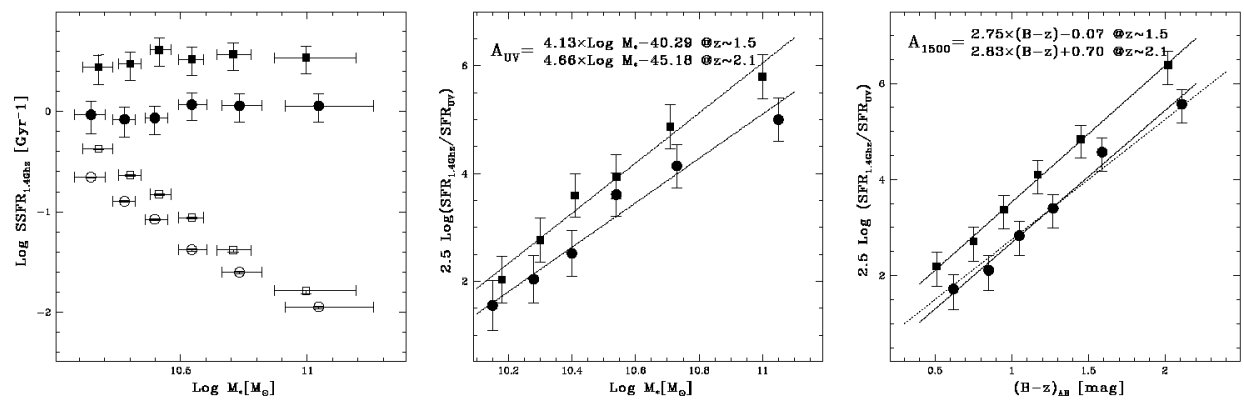

FIGURE 4. Left: Radio derived specific star formation rate (SSFR, solid symbols) compared with the uncorrected UV derived SSFR (empty symbols) in two redshift bins ( $\mathrm{z} \approx 1.5$ - circles and $\mathrm{z} \approx 2.1$ - squares), as a function of $\log M_{*}$. Middle: UV light attenuation as a function of galaxy stellar mass. Right: UV light attenuation as a function of B-z color (UV slope). The dotted line shows the attenuation law derived in [7] following the Calzetti law [13] prescription.

as $U$ dropouts" [14]. In the middle/right panels of Fig. 4 we provide the UV light attenuation as a function of both stellar mass and UV slope: this implies a well defined mass-metallicity/dust relation in place at $z \approx 2$. The dust attenuation at $1500 \AA$ we found is empirical, model independent and also in good agreement with the Calzetti law [13].

\section{ACKNOWLEDGMENTS}

The National Radio Astronomy Observatory is a facility of the National Science Foundation operated under cooperative agreement by Associated Universities, Inc. The HST COSMOS Treasury program was supported through NASA grant HST-GO- 09822. We gratefully acknowledge the contributions of the entire COSMOS collaboration consisting of more than 100 scientists (http://www.astro.caltech.edu/cosmos).

\section{REFERENCES}

1. N. Drory, M. Salvato, A. Gabasch et al., ApJL 619, L131 (2005).

2. M. S. Yun, N. A. Reddy, and J. J. Condon, $A p J$ 554, 803 (2001).

3. L. L. Cowie, A. Songaila, E. M. Hu, J. G. Cohen, AJ 112, 839 (1996).

4. M. Franx, P. G. van Dokkum, N. M. F. Schreiber et al., ApJ 688, 770 (2008).

5. E. Schinnerer, V. Smolčić, C. L. Carilli et al., ApJS 172, 46 (2007).

6. P. Capak, H. Aussel, M. Ajikiet al., ApJS 172, 99 (2007).

7. E. Daddi, A. Cimatti, A. Renzini et al., ApJ 617, 746 (2004).

8. E. E. Salpeter, $A p J 121,161$ (1995).

9. V. Smolčić, E. Schinnerer, M. Scodeggio et al., ApJS 177, 14 (2008).

10. O. Ilbert, P. Capak, M. Salvato et al., ArXiv e-prints (2008), 0809.2101.

11. M. Salvato, G. Hasinger, O. Ilbert et al., ArXiv e-prints (2008), 0809.2098.

12. L. Dunne, R. J. Ivison, S. Maddox et al, ArXiv e-prints (2008), 0808.3139.

13. D. Calzetti, A. L. Kinney and T. Storchi-Bergmann, ApJ 429, 582 (1994).

14. G. R Meurer, T. M. Heckman and D. Calzetti, ApJ 521, 64 (1999). 